\title{
Evaluation of the Parameters Affecting the Success Rate of the Microfluidics-Based Sperm Selection for In Vitro Fertilization
}

\author{
Tolga Ecemiş ${ }^{1 *}$, Gamze Bilgili ${ }^{2}$ \\ ${ }^{1}$ Ecemis Klinik, Ankara, Turkey \\ ${ }^{2}$ Department of Physiology, Gazi University, Ankara, Turkey
}

*Corresponding Author: Tolga Ecemiş, Ecemis Klinik Çukurambar Mah. 1480 Sok. Besakule A Blok Kat:11 No:1/41 Çankaya, Ankara, Turkey.

\begin{abstract}
Introduction: Recently, microfluidic-based approaches have been proposed to determine and obtain the finest quality sperm before in vitro fertilization (IVF) process. In the present study, we aimed to report the IVF outcomes where a microfluidics-based chip system was used for obtaining the sperm with top-quality.
\end{abstract}

Material and Methods: The IVF success of the patients with male factor infertility whose semen analyses were obtained by seminograms and to whom microfluidics-based sperm sorting was applied was retrospectively evaluated by emphasizing the criteria that may affect the success rate.

Results: The results showed that the system was able to choose the appropriate sperm for even the total number of the sperm (400 thousand compared to the reference 33-46 million) and the ratio of the quality sperm motility, and morphology (2\% of them morphologically normal) were very low among the sperm populations. In addition, the infertility duration significantly affected the success rate of IVF via chip-based sperm sorting.

Conclusions: These results underline the success ratio of the designed microfluidic system in the IVF outcome, emphasizing again the importance of sperm quality in IVF processes and guide the clinicians to apply this procedure especially to the patients diagnosed with infertility for short time periods.

Abbreviations: IVF: in vitro fertilization, IUI: intrauterine insemination, ICSI: intracytoplasmic sperm injection

Key Message: Duration of infertility in men affect the success rate of the microfluidics-based sperm sorting.

Keywords: Sperm sorting, infertility of men, microfluidics, in vitro fertilization, duration of fertility

\section{INTRODUCTION}

Infertility has nowadays been one of the major reproductive health problems worldwide [1]. The advances in the technology such as in vitro fertilization (IVF), intrauterine insemination (IUI) and intracytoplasmic sperm injection (ICSI) are the fundamental approaches to overcome the infertility problem [2].

The developed technologies for the treatment of infertility have frequently been shown to be affected by several factors including woman age, cause of infertility, levels of follicle stimulating hormone, adequate ovarian responses and number of collected eggs [3]. Moreover, sperm quality has also been underlined to be responsible for the failure of the IVF successes [4], and male factor was clarified to contribute IVF failure in a rate of 30$50 \%$ [5]. Sperm morphology, motility, count and genomic integrity have been figured out to be criteria affecting sperm quality and some methods to test and choose the sperm with high quality have been developed [6]. Among these methods, density gradient and swim-up techniques by which high quality sperms are obtained by centrifugation and via endogenous sperm motility property, respectively have widely been used in the clinics. However, these methods could be disadvantageous, as they would result in low sperm number and/or loss in sperm quality [7].

Recently, microfluidics-based novel approaches have been proposed for testing and obtaining the 
sperm with high quality for IVF procedure. The microfluidics-based devices use the ability of endogenous or directed motility of the sperm and not only choose the sperms with high motility properties but also enables further morphological and genomic analyses by minimizing the damages on the selected sperms [8-9].

Although the efficacy and the sufficiency of the developed devices for the sperm selection has been studied during the production period [9], clinical reflections of these devices must be followed for further progress and identification of possible drawbacks. For instance, Yildiz and Yuksel studied the efficacy of the sperm selection by a microfluidics-based chip (Fertile Plus; Koek Biotechnology, Turkey) for 116 patients and compared the results to that of known density gradient method via fertilization rates. The chip was demonstrated to increase fertilization rate especially in recurrent IVF failure patients by decreasing sperm DNA fragmentation [10]. However, such studies are still needed to totally explore the success rate of chip-based sperm sorting for IVF procedure.

In the present study, we evaluated the efficacy of a microfluidic chip for the sperm selection (Fertile Plus; Koek Biotechnology, Turkey) for 57 couples. Moreover, we analysed the positive and negative pregnancies by correlating to the sperm number, sperm motility, sperm morphology and fertilization trials.

\section{MATERIALS AND METHODS}

\subsection{Patient Selection}

The present study covers the evaluation of microfluidics-based sperm sorting for 50 couples between 2017-2018 in HRS Ankara Women's Hospital, Ankara, Turkey. The retrospective evaluation eliminated the requirement of ethics committee approval; however, patients' signed approvals have been obtained. The semen quality was assessed by standard seminograms and used without further evaluation.

\subsection{Preparation of Sperms with Microchip Method}

For sperm sorting with microfluidics-based sperm chip, Fertile Plus Chip (Koek Biotechnology, Turkey) was used according to supplier's instructions. Briefly, sperm samples were added onto chip as $800 \mu$ of the volume and washed by $1 \mathrm{ml}$ of sperm washing solution.
Then, the chip was incubated at $37^{\circ} \mathrm{C}$ for $30 \mathrm{~min}$ to obtain the sperms passing through the filter and localizing in the washing medium. Next, the sperms obtained by this protocol were used for the IVF protocol.

\subsection{Statistical Analyses}

The normality of the data was analysed by Kolmogorov-Smirnov normality test. The data was analysed by Mann-Whitney $U$ test as a result of lack of normality. All analyses were carried out by GraphPad Prism (GraphPad Inc., USA) and the data was significant when $\mathrm{p}<0.05$.

\section{Results}

We listed the parameters as age of female and male, reason of infertility whether it was primer or seconder, total duration of infertility, the number of IVF trials, sperm number, sperm motility (\%), sperm morphology (\%), the number of oocytes used for fertilization, the number of the metaphase II which demonstrated the increased chance of fertilization rate [11], the number of fertilizations, embryo transfer method whether directly (ET) or after freezethaw cycle (FET) and the status of beta HCG that illustrated the availability of ectopic pregnancy [12] by emphasizing the cases whether positive, negative, terminated pregnancy (Ex) or early birth (EB) in a group of couples $(n=50)$ with infertility reason of male factor (Table 1).The results illustrated that the microfluidics-based sperm sorting was highly effective to obtain beta HCG positivity even though the per cents of sperm motility and/or morphology were extremely low, clarifying that the system chose the sperms with high quality independent of sperm morphology and/or motility criteria.

We further analyzed the sperm motility which was the major factor affecting the working principle of the sperm chip. The results were given at Table 2 where the motilities were given as per cents according to the classification of the sperm motility as grade a, b, c or d that shows rapidly progressive, slowly progressive, no progressive and immotile spermatozoa, respectively [13]. Nevertheless, there were not any significance between beta HCG positive and negative groups in terms of sperm motility, pointing that the motility of sperms was not a critical factor affecting the efficacy of the sperm sorting by microfluidic approach (Table 3). 
Table1. The list of patients and sperm profiles with the result of beta CGH status

\begin{tabular}{|c|c|c|c|c|c|c|c|c|c|c|c|c|c|}
\hline Patients & $\begin{array}{l}\text { Age of } \\
\text { female }\end{array}$ & $\begin{array}{c}\text { Age of } \\
\text { male }\end{array}$ & $\begin{array}{l}\text { Reason of } \\
\text { infertility }\end{array}$ & $\begin{array}{c}\text { Year of } \\
\text { infertility }\end{array}$ & $\begin{array}{c}\text { Number of } \\
\text { trials }\end{array}$ & $\begin{array}{c}\text { Sperm } \\
\text { number }\end{array}$ & \begin{tabular}{|c|} 
Sperm \\
motility $(\%)$
\end{tabular} & \begin{tabular}{|c|} 
Sperm \\
morphology $(\%)$
\end{tabular} & \begin{tabular}{|c|} 
Oocyte \\
number
\end{tabular} & $\begin{array}{c}\text { Number of } \\
\text { Metaphase II }\end{array}$ & $\begin{array}{c}\text { Number of } \\
\text { fertilization }\end{array}$ & Transfer & $\begin{array}{c}\text { Beta } \\
\text { HCG (+/-) }\end{array}$ \\
\hline 1 & 33 & 33 & Primer & 3 & 2 & $31 \times 10^{6}$ & 60 & 5 & 7 & 5 & 5 & FET & + \\
\hline 2 & 38 & 42 & Primer & 2 & 1 & $63 \times 10^{6}$ & 62 & 0 & 8 & 7 & 5 & ET & - \\
\hline 3 & 40 & 42 & Primer & 12 & 8 & $3 \times 10^{6}$ & 20 & 0 & 8 & 7 & 5 & FET & - \\
\hline 4 & 39 & 43 & Seconder & 15 & 1 & $44 \times 10^{6}$ & 18 & 0 & 12 & 9 & 6 & FETx2 & + \\
\hline 5 & 36 & 42 & Primer & 2 & 3 & $4 \times 10^{6}$ & 30 & 0 & 7 & 6 & 3 & FET & + \\
\hline 6 & 34 & 34 & Primer & 1.5 & 3 & $4 \times 10^{5}$ & 2 & 0 & 20 & 16 & 9 & FETx5 & + \\
\hline 7 & 27 & 28 & Primer & 4 & 2 & $10 \times 10^{6}$ & 70 & 1 & 7 & 5 & 5 & ET & - \\
\hline 9 & 41 & 42 & Seconder & 9 & 6 & $44 \times 10^{6}$ & 45 & 0 & 3 & 3 & 2 & ET & - \\
\hline 10 & 29 & 32 & Primer & 1 & 1 & $120 \times 10^{6}$ & 49 & 3 & 14 & 11 & 8 & FET & + \\
\hline 11 & 31 & 31 & Primer & 5 & 2 & $32 \times 10^{6}$ & 65 & 3 & 12 & 8 & 7 & ET & + \\
\hline 12 & 24 & 24 & Primer & 2 & 1 & $216 \times 10^{6}$ & 69 & 2 & 15 & 13 & 11 & FET & + \\
\hline 13 & 37 & 37 & Primer & 6 & 10 & $10 \times 10^{6}$ & 52 & 0 & 3 & 2 & 1 & FET & + \\
\hline 14 & 31 & 36 & Primer & 2 & 2 & $12 \times 10^{6}$ & 42 & 0 & 13 & 10 & 7 & FET & Ex \\
\hline 15 & 27 & 32 & Primer & 1 & 2 & $2 \times 10^{6}$ & 37 & 0 & 15 & 9 & 6 & FET & + \\
\hline 16 & 25 & 27 & Primer & NA & 1 & $8 \times 10^{5}$ & 36 & 0 & 11 & 9 & 9 & ET & - \\
\hline 18 & 34 & 37 & Primer & 9 & 7 & $4 \times 10^{6}$ & 58 & 0 & 10 & 9 & 6 & FET & - \\
\hline 19 & 33 & 37 & Primer & NA & 1 & $37 \times 10^{6}$ & 60 & 0 & 14 & 9 & 7 & ET & - \\
\hline 20 & 40 & 42 & Seconder & 1 & 1 & $72 \times 10^{6}$ & 77 & 0 & 5 & 5 & 4 & FETx2 & + \\
\hline 21 & 34 & 34 & Primer & NA & NA & $43 \times 10^{6}$ & 74 & 4 & 17 & 16 & 13 & FET & + \\
\hline 22 & 25 & 25 & Primer & 2 & 1 & $42 \times 10^{6}$ & 74 & 0 & 13 & 11 & 8 & FET & + \\
\hline 23 & 27 & 27 & Primer & 3 & 1 & $34 \times 10^{6}$ & 56 & 0 & 11 & 8 & 6 & FET & + \\
\hline 24 & 27 & 28 & Primer & 4 & 3 & $5 \times 10^{6}$ & 72 & 0 & 12 & 11 & 11 & FET & - \\
\hline 25 & 35 & 36 & Primer & 1 & 1 & $108 \times 10^{6}$ & 70 & 0 & 5 & 3 & 3 & ET & + \\
\hline 26 & 27 & 28 & Seconder & 2 & 1 & $73 \times 10^{6}$ & 52 & 1 & 12 & 11 & 9 & FET & EB \\
\hline 27 & 23 & 23 & Primer & 3 & 1 & $47 \times 10^{6}$ & 63 & 0 & 16 & 14 & 10 & FET & - \\
\hline 28 & 40 & 40 & Seconder & $?$ & 6 & $154 \times 10^{6}$ & 73 & 4 & 2 & 1 & 1 & ET & - \\
\hline 29 & 38 & 139 & Primer & 0.5 & 1 & $87 \times 10^{6}$ & 72 & 1 & 2 & 1 & 1 & ET & - \\
\hline 30 & 32 & 33 & Primer & 1.5 & 1 & $47 \times 10^{6}$ & 74 & 1 & 14 & 11 & 11 & ET & + \\
\hline 31 & 35 & 35 & Primer & 15 & 2 & $145 \times 10^{6}$ & 82 & 0 & 6 & 5 & 4 & ET & Ex \\
\hline 32 & 28 & 29 & Primer & 2 & 2 & $69 \times 10^{6}$ & 52 & 0 & 14 & 10 & 7 & FET & + \\
\hline 33 & 34 & 35 & Seconder & 1 & 2 & $50 \times 10^{6}$ & 70 & 0 & 12 & 11 & 9 & ET & + \\
\hline 34 & 35 & 36 & Primer & 1 & 1 & $2 \times 10^{6}$ & 27 & 0 & 14 & 11 & 8 & ET & + \\
\hline
\end{tabular}


Evaluation of the Parameters Affecting the Success Rate of the Microfluidics-Based Sperm Selection for In Vitro Fertilization

\begin{tabular}{|c|c|c|c|c|c|c|c|c|c|c|c|c|c|}
\hline 35 & 35 & 35 & Primer & 2 & 2 & $1 \times 10^{6}$ & 47 & 0 & 12 & 10 & 10 & FET & - \\
\hline 36 & 40 & 43 & Primer & 3 & 4 & $9 \times 10^{6}$ & 12 & 2 & 4 & 2 & 2 & FET & - \\
\hline 37 & 37 & 38 & Primer & 1 & 1 & $64 \times 10^{6}$ & 70 & 0 & 12 & 9 & 6 & ET & - \\
\hline 38 & 30 & 33 & Primer & 3 & 3 & $330 \times 10^{6}$ & 63 & 2 & 11 & 6 & 6 & ET & + \\
\hline 39 & 39 & 43 & Primer & 18 & 3 & $15 \times 10^{6}$ & 86 & 0 & 10 & 7 & 7 & ET & - \\
\hline 40 & 32 & 32 & Seconder & 2 & 3 & $32 \times 10^{6}$ & 47 & 0 & 11 & 9 & 9 & FET & + \\
\hline 41 & 29 & 33 & Primer & 5 & 3 & $16 \times 10^{6}$ & 30 & 0 & 10 & 8 & 7 & FET & + \\
\hline 42 & 26 & 28 & Primer & 2 & 1 & $22 \times 10^{6}$ & 56 & 0 & 6 & 5 & 5 & FET & + \\
\hline 43 & 37 & 37 & Primer & 1 & 2 & $105 \times 10^{6}$ & 72 & 0 & 6 & 6 & 6 & FET & + \\
\hline 45 & 24 & 28 & Primer & 2 & 2 & $150 \times 10^{6}$ & 43 & 0 & 13 & 10 & 9 & FET & + \\
\hline 46 & 30 & 30 & Primer & 2 & 2 & $68 \times 10^{6}$ & 48 & 0 & 16 & 14 & 14 & FET & + \\
\hline 47 & 33 & 33 & Primer & 3 & 2 & $44 \times 10^{6}$ & 31 & 0 & 6 & 5 & 4 & FET & - \\
\hline 48 & 34 & 34 & Primer & 2 & 1 & $115 \times 10^{6}$ & 74 & 0 & 29 & 26 & 22 & FET & $\mathrm{Ex}$ \\
\hline 49 & 28 & 28 & Primer & 1.5 & 1 & $280 \times 10^{6}$ & 43 & 3 & 16 & 13 & 10 & FET & + \\
\hline 50 & 33 & 33 & Primer & 1 & 1 & $21 \times 10^{6}$ & 42 & 0 & 11 & 10 & 10 & ET & + \\
\hline
\end{tabular}


Evaluation of the Parameters Affecting the Success Rate of the Microfluidics-Based Sperm Selection for In Vitro Fertilization

Amongst the parameters given above, age of female or male, sperm number, motility and morphology, and metaphase II and fertilization number did not significantly deviate between beta HCG negative and positive groups.
However, the year of infertility was significantly $(p<0.05)$ lower and the number of oocytes used for IVF procedure was significantly higher in beta HCG positive group compared to negative one (Table 3 ).

Table2. Sperm motilities as per cent of the motility grades for beta HCG positive and negative groups

\begin{tabular}{|c|c|c|c|c|c|c|c|c|}
\hline & \multicolumn{9}{|c|}{ Beta HCG (+) } & \multicolumn{3}{c|}{ Beta HCG (-) } \\
\hline Patients & a & b & c & Total & a & b & c & Total \\
\hline 1 & 7 & 33 & 20 & 60 & - & 46 & 16 & 62 \\
\hline 2 & - & 14 & 4 & 18 & - & 4 & 16 & 20 \\
\hline 3 & - & 22 & 8 & 30 & 4 & 55 & 11 & 70 \\
\hline 4 & - & 1 & 1 & 2 & NA & NA & NA & NA \\
\hline 5 & 6 & 29 & 14 & 49 & - & 33 & 12 & 45 \\
\hline 6 & 5 & 50 & 10 & 65 & - & 24 & 12 & 36 \\
\hline 7 & 6 & 56 & 7 & 69 & - & 52 & 6 & 58 \\
\hline 8 & - & 39 & 13 & 52 & - & 48 & 12 & 60 \\
\hline 9 & - & 30 & 7 & 37 & - & 68 & 4 & 72 \\
\hline 10 & - & 48 & 4 & 52 & - & 55 & 8 & 63 \\
\hline 11 & 9 & 56 & 12 & 77 & 8 & 55 & 10 & 73 \\
\hline 12 & 10 & 56 & 8 & 74 & 3 & 55 & 14 & 72 \\
\hline 13 & - & 58 & 16 & 74 & - & 30 & 17 & 47 \\
\hline 14 & - & 46 & 10 & 56 & - & 10 & 2 & 12 \\
\hline 15 & 6 & 47 & 17 & 70 & - & 58 & 12 & 70 \\
\hline 16 & - & 40 & 12 & 52 & - & 79 & 7 & 86 \\
\hline 17 & - & 63 & 7 & 70 & - & 23 & 8 & 31 \\
\hline 18 & - & 17 & 10 & 27 & & & & \\
\hline 19 & 10 & 32 & 21 & 63 & & & & \\
\hline 20 & - & 38 & 9 & 47 & & & & \\
\hline 21 & - & 27 & 3 & 30 & & & & \\
\hline 22 & - & 46 & 10 & 56 & & & & \\
\hline 23 & - & 48 & 24 & 72 & & & & \\
\hline 24 & - & 42 & 26 & 68 & & & & \\
\hline 25 & - & 21 & 27 & 48 & & & & \\
\hline 26 & - & 37 & 6 & 43 & & & & \\
\hline 27 & - & 30 & 12 & 42 & & & & \\
\hline Mean & 2.18 & 38.00 & 11.78 & 52.78 & 0.88 & 43.44 & 10.44 & 54.78 \\
\hline & & & & & & & & \\
\hline
\end{tabular}

Table3. The parameters that may affect the beta HCG status and their significance

\begin{tabular}{|c|c|c|c|}
\hline Parameters & Beta HCG (+) & Beta HCG (-) & p value \\
\hline Number of couples & 27 & 17 & 0.31 \\
\hline Age of female & $32 \pm 0.81$ & $34 \pm 1.41$ & 0.22 \\
\hline Age of male & $33 \pm 0.91$ & $35 \pm 1.50$ & $0.02^{*}$ \\
\hline $\begin{array}{c}\text { Reason of infertility (Number of } \\
\text { seconder/primer) }\end{array}$ & $5 / 22$ & $2 / 15$ & 0.27 \\
\hline Year of infertility & $2.67 \pm 0.56$ & $5.30 \pm 1.32$ & 0.74 \\
\hline Sperm number & $70 \times 10^{6} \pm 16 \times 10^{6}$ & $38 \times 10^{6} \pm 10 \times 10^{6}$ & 0.27 \\
\hline Total sperm motility (\%) & $52.78 \pm 3.72$ & $54.78 \pm 5.25$ & 0.30 \\
\hline Grade a sperm motility (\%) & $2.18 \pm 0.68$ & $0.88 \pm 0.52$ & 0.86 \\
\hline Grade b sperm motility (\%) & $38.00 \pm 2.89$ & $43.44 \pm 5.19$ & 0.52 \\
\hline Grade c sperm motility (\%) & $11.78 \pm 1.33$ & $10.44 \pm 1.08$ & $0.05^{*}$ \\
\hline Sperm morphology (\%) & $0.85 \pm 0.28$ & $0.85 \pm 0.28$ & 0.09 \\
\hline Oocyte number & $11.63 \pm 0.87$ & $8.70 \pm 1.02$ & 0.11 \\
\hline Number of Metaphase II & $9.29 \pm 0.79$ & $6.94 \pm 0.88$ & $5.76 \pm 0.75$ \\
\hline Number of fertilizations & $7.55 \pm 0.63$ & & \\
\hline
\end{tabular}




\section{DisCUSSION}

In the present retrospective study, we evaluated the cases where the couples faced with primer or seconder infertility because of the male factors. The sperm quality was assessed by a microfluidic-based sperm chip, Fertile Chip [9] in parallel to the conventional semen analysis (seminogram). We reviewed the parameters that could affect the fertility in Table 1 and tried to examine these parameters in parallel to the success rate of the microfluidics-based sperm sorting approach.

Aafjes et al. underlined that the duration of infertility was a critical factor affecting the prognosis of the fertility in men and increased time stimulated the semen abnormalities [14]. In parallel to this report, the microfluidics-based sperm sorting was successful in terms of the selection of the sperms with higher quality since the short duration of fertility resulted in higher rate of positive beta HCG by Fertile Sperm Chip.

According to the comparative analyses, the number of oocytes was significantly higher in beta HCG positive group, pointing more oocyte is advantageous in terms of obtaining positive results for IVF procedure via microchip-based sperm sorting. However, the number of metaphase II did not deviate significantly between groups, eliminating the hypothesis of that high number of oocytes are needed. Additionally, the number oocytes with metaphase II was also not fundamental for IVF procedure depending on microchip-based sperm sorting.

\section{CONClusion}

In vitro fertilization and embryo transfer (IVFET) has been an acceptable technique for the treatment of certain infertility cases. The outcomes of IVF-ET process have frequently been shown to be affected by several critical factors, one of which was sperm motility and morphology. Therefore, in addition to other fundamental requirements, sperm motility and morphology are amongst the criteria that should be taken into consideration during IVF process. Sperm quality has been proposed to be tested by several methods including microfluidics-based sperm sorting. In the present study, we retrospectively evaluated the parameters affecting the IVF success rate via microfluidicsbased sperm sorting. Our results showed that this approach was successful even the per cent of the sperm motilities and/or morphologies were extremely low. In addition, the duration of men fertility was the only criterion significantly affecting the IVF outcome for microfluidicsbased sperm sorting. These results could guide the clinicians to apply microfluidics-based sperm sorting for IVF particularly in the couples with short infertility durations.

\section{ACKNOWLEDGEMENT}

We would like to thank the patient approving the usage of the test results.

\section{DISCLOSURES}

All authors declare that they have no conflict of interest.

This article does not contain any studies with animal subjects performed by the any of the authors. The retrospective evaluation eliminated the requirement of ethics committee approval; however, patients' signed approvals have been obtained.

\section{REFERENCES}

[1] Inhorn MC, Patrizio P. Infertility around the globe: new thinking on gender, reproductive technologies and global movements in the $21 \mathrm{st}$ century. HumReprod. 2015;21(4):411-426.

[2] Mascarenhas MN, Flaxman SR, Boerma T, Vanderpoel S, Stevens GA. National, regional and globaltrends in infertility prevalence since 1990: a systematic analysis of 277 health surveys. PLoSMed.2012;9(12):e1001356.

[3] Qublan HS, Malkawi HY, Tahat YA, Areidah S, Nusair B, Kheisat BM, Al-Quraan G, AbuAssaf A, Hadaddein MF, Abu-Jassar H. In vitro fertilization treatment: Factors affecting its results and outcome. J ObstetGynaecol. 2005;7:689-693.

[4] Donnelly ET, Lewis SE, McNally JA, Thompson W. In vitro fertilization and pregnancy rates: the influence of sperm motility and morphology on IVF outcome. Fertil Steril. 1998;70(2): 305-314.

[5] Boivin J, Bunting L, Collins JA, Nygren KG. International estimates of infertility prevalence and treatment-seeking: potential need and demand for infertility medicalcare. Hum Reprod. 2007;22(6):1506-12.

[6] Tanphaichitr N, Agulnick A, Seibel M, Taymor M. Comparison of the in vitro fertilization rate byhuman sperm capacitated by multipletubeswim-up and Percollgradient centrifugation. J InVitro Fert EmbryoTransf. 1988;5(3):119-22. 
[7] Aitken RJ, Clarkson JS. Significance of reactive oxygen species and antioxidants in defining the efficacy of sperm preparation techniques. J Androl. 1988;9(6):367-76.

[8] Knowlton SM, Sadasivam M, Tasoglu S. Microfluidics for sperm research. Trends Biotechnol. 2015;33(4):221-229.

[9] Yetkinel S, Kilicdag EB, Aytac PC, Haydardedeoglu B, Simsek E, Cok T. Effects of the microfluidic chip technique in sperm selection for intracytoplasmic sperm injection for unexplained infertility: a prospective, randomized controlled trial. J AssistReprodGenet. 2019;36(3):403-409.

[10] Yildiz K, Yuksel S. Use of microfluidic sperm extractionchips as an alternative method in patients with recurrent in vitro fertilisation failure. J AssistReprodGenet. 2019:1-7.
[11] De Vos A, Van de Velde H, Joris H, Van Steirteghem A. In-vitro matured metaphase-I oocytes have a lower fertilization rate but similar embryo quality as mature metaphase-II oocytes after intracytoplasmic sperm injection. Hum Reprod. 1999;14(7):1859-1863.

[12] Schwartz RO, Di DP. Beta-hCG as a diagnostic aid for suspected ectopic pregnancy. ObstetGynecol. 1980;56(2):197203.

[13] Nabi A, Khalili MA, Halvaei I, Roodbari F. Prolonged incubation of processed human spermatozoa will increase DNA fragmentation. Andrologia. 2014;46:374-9.

[14] Aafjes JH, vdVijver JC, Schenck PE. The duration of infertility: an important datum for the fertility prognosis of men with semen abnormalities. FertilSteril. 1978;30(4):423-425.

Citation: Tolga Ecemiş, Gamze Bilgili. Evaluation of the Parameters Affecting the Success Rate of the Microfluidics-Based Sperm Selection for In Vitro Fertilization. ARC Journal of Clinical Case Reports. 2020; 6(3):6-12. DOI: https://doi.org/ 10.20431/2455-9806.0603002.

Copyright: (C) 2020 Authors. This is an open-access article distributed under the terms of the Creative Commons Attribution License, which permits unrestricted use, distribution, and reproduction in any medium, provided the original author and source are credited. 